\title{
Bird richness and composition in a Cerrado fragment in the State of São Paulo
}

\author{
Manica, LT.*, Telles, M. and Dias, MM. \\ Departamento de Ecologia e Biologia Evolutiva, \\ Universidade Federal de São Carlos - UFSCar \\ CEP 13565-905, São Carlos, SP, Brazil \\ *e-mail: lilianmanica@gmail.com
}

Received September 5, 2008 - Accepted December 23, 2008 - Distributed May 31, 2010

\begin{abstract}
Bird species richness is an important measure for monitoring biodiversity changes. We analysed avifauna richness and composition in a 472 ha protected cerrado fragment and surroundings at Fazenda Canchim (RL-CPPSE), São Carlos, in the State of São Paulo (SP). We carried out 95.1 hours of observation (22 visits) at irregular intervals from May 2004 to December 2006. Qualitative surveys were done walking through tracks inside the fragment and on the roads at its edge. We recorded 160 species, six of which were endemic to Cerrado domain, 22 migratory, seven threatened within the State of São Paulo, and two globally threatened. We found 28 species in the cerradão, 110 in the cerrado sensu stricto, 13 in the gallery forest, 26 in the reservoir border, 26 in pasturelands and sugar cane monoculture and 55 in an anthropic area. Most of the species had low frequency of occurrence in all vegetation forms. Insectivores were the major trophic category (46.9\%), which is typical in tropical regions, and it is also related to resource availability. Omnivores followed with $19.4 \%$, granivores with $8.8 \%$ and frugivores with $7.5 \%$. We conclude that, despite its size and conservation status, our study area has a remarkable bird community and must be considered as a priority conservation area to preserve bird species in Sao Paulo State.
\end{abstract}

Keywords: avifauna, State of São Paulo, Cerrado, fragmentation.

\section{Riqueza e composição da avifauna em um fragmento de Cerrado no Estado de São Paulo}

\begin{abstract}
Resumo
A riqueza de espécies é uma medida importante para o monitoramento da perda de biodiversidade. Nosso objetivo foi verificar a riqueza e a composição da avifauna em um fragmento de cerrado de 472 ha na Fazenda Canchim (RLCPPSE), São Carlos, Estado de São Paulo (SP). Realizamos 95,1 horas de observação (22 visitas) entre maio de 2004 e dezembro de 2006, em intervalos irregulares. Percorremos trilhas dentro do fragmento e estradas na sua borda considerando diferentes tipos de vegetação. Registramos 160 espécies, das quais seis são endêmicas do Cerrado, 22 migratórias, sete ameaçadas de extinção no estado de São Paulo e duas globalmente ameaçadas. Registramos 28 espécies no cerradão, 110 no cerrado sensu stricto, 13 na mata ciliar, 26 no entorno de uma represa, 26 em pastagens e plantações de cana-de-açúcar e 55 em áreas antropizadas. A maioria das espécies ocorreu em baixa frequiência em todos os tipos de vegetação. A categoria com maior número de espécies foi a dos insetívoros (46,9\%), padrão típico em regiões tropicais e, provavelmente, relacionado à disponibilidade de recursos na área. A segunda categoria trófica foi a dos onívoros $(19,4 \%)$, seguida pelos granívoros $(8,8 \%)$ e frugívoros $(7,5 \%)$. Nossos resultados destacam consideráveis registros de espécies aves e a importância da área de estudo na preservação da biodiversidade da avifauna no cerrado paulista.
\end{abstract}

Palavras-chave: avifauna, Estado de São Paulo, Cerrado, fragmentação.

\section{Introduction}

Bird species richness is an important measure for monitoring biodiversity changes (Andrén, 1994; Palmer et al., 2008). Bird community inventories, censuses and surveys provide data of great value for conservation purposes (e.g. Tubelis et al., 2004; Marini and Garcia, 2005;

Silva, 1997). For instance, these methods allow the recognition of endemic, threatened, keystone, umbrella or surrogate group of species. Because of their restricted distribution, vulnerability, high influence on other species occurrence or important ecological role in ecosystems, 
registration of these species might strongly influence priority activities in the protection of the natural environment (Stotz et al., 1996; Silva and Bates 2002, Loyola et al., 2007; Pinto et al., 2008, Piratelli et al., 2008).

The study of bird species richness also improves the knowledge of community responses in disturbed areas, because richness may be reduced in long-time isolated fragments (Aleixo and Vielliard, 1995; Marini, 2001; Stouffer and Bierregaard, 1995). Small remnants are prone to edge effects, such as nest predation increase (Marini et al., 1995), attraction of exotic predators, diseases and competitors or migratory and dispersal route obstructions (review in Marzluff and Ewing, 2001). All these factors may affect individual chances of reproduction and reduce local species richness (Andrén, 1994).

In addition, bird richness and composition offers valuable data to understand sensitivity and resistance of species to fragmentation (Zanette et al., 2000). Speciesspecific responses to fragmentation are widely diverse among birds (Debinski and Holt, 2000; Watson et al., 2005). For example, local migratory birds and species that need specific nest sites may require extensive areas to find environmental conditions to survive and breed (Sick, 1997). Furthermore, fragmentation may influence the trophic structure of bird communities (Motta-Junior, 1990; Tubelis and Cavalcanti, 2000). For example, specialist groups, such as understory insectivores, are negatively affected in fragmented landscapes because of the reduced suitable pathways connecting multiple foraging areas (Martensen et al., 2008).

Agriculture and pastureland expansions in the Cerrado domain - one of the global biodiversity hotspots (Myers et al., 2000) - have been the major threatening factors to its avifauna biodiversity since the 1970s (Alho and Martins, 1995; Cavalcanti and Joly, 2002). The Cerrado domain has 837 bird species (Silva, 1995), from which $90.7 \%$ breed within the domain and the others migrate from the Northern Hemisphere, southern South America and southeast Brazil (Silva, 1995; 1997). Endemism in the region is high, but almost $75 \%$ of endemic cerrado avifauna is considered at risk (Stotz et al., 1996). Furthermore, although endemic bird areas occur in the Cerrado (Birdlife International, 2003), 20\% of endemic and threatened species are outside protected areas (Machado et al., 2004).

In the State of São Paulo, the Cerrado has been reduced to less than $7 \%$ of its original cover for the last decades and only $18 \%$ of remnants are inside protected areas (São Paulo, 1999). Different from conservation areas of other domains, few Cerrado fragments are greater than 400 ha (Durigan et al., 2003). Considering the high level of degradation of the Cerrado in São Paulo, and regarding its boundary with the Atlantic Forest domain, these remnants should be concerned as important areas for conservation plans (Cavalcanti and Joly, 2002).

Even though some studies of bird communities have been previously carried out in São Paulo (Motta-Junior, 1990; Dias, 2000; Willis and Oniki, 2003; Willis, 2004;
Develey et al., 2005; Willis, 2006; Motta-Junior et al., 2008), little is known about the ecology of bird communities in the region. Our aim was to contribute with the knowledge of the avifauna of one fragment in central São Paulo and give data support for future local or regional reaseaches. We studied bird species richness and composition, and identified endemic, threatened and migratory species of a protected cerrado fragment at Fazenda Canchim (RL-CPPSE), São Carlos, SP.

\section{Material and Methods}

Our study site is a 472 ha protected cerrado fragment at Fazenda Canchim (Reserva Legal-Embrapa Pecuária Sudeste, RL-CPPSE) and its surroundings, $12 \mathrm{~km}$ from São Carlos, in the State of São Paulo (SP) (21 ${ }^{\circ} 55^{\prime}$ S and $47^{\circ} 48^{\prime} \mathrm{W}$ ) (Primavesi et al., 1999). This is a transition zone from the Atlantic Forest to Cerrado domain, also characterised as an ecological tension zone, and with a high level of biodiversity (Primavesi et al., 1999). The climate is typical of moist savanna (Cwa, according to Köppen's classification), with a remarkable seasonality: a dry and cold winter from April to September and a wet and warmer summer from October to March (Primavesi et al., 1999).

The reserve is covered by two cerrado physiognomies: cerrado sensu stricto (340 ha), a typical savanna vegetation, and cerradão (130 ha), an almost closed woodland. A gallery forest (1.35 ha) occurs along a stream (Córrego da Lagoa) inside the fragment. The surroundings are composed of a reservoir (Represa do 29 ) in the north-east of the reserve and between cerrado sensu stricto and a small village; a 120 ha cerrado fragment in the east; pasturelands in the south and south-east and sugar cane monoculture in the north and west of the reserve (Primavesi et al., 1999).

We did qualitative surveys during mornings, from 06:00 to 11:00 AM, and late-afternoon, from 03:00 to 06:00 PM, in 95.1 hours of observation in irregular intervals from 2004 to 2006. The area was visited 22 times: six in 2004 (May, August, November and December), 14 in 2005 (from January to December, except in March, July and August) and two in 2006 (January and December). We sampled all vegetation forms described above walking through $15 \mathrm{~km}$ trails or roads, approximately: 1) $3.8 \mathrm{~km}$ trail inside the fragment $(1.55 \mathrm{~km}$ in cerrado sensu stricto, $1.25 \mathrm{~km}$ in gallery forest and $1 \mathrm{~km}$ in cerradão); 2) $2 \mathrm{~km}$ road in the middle of the reserve through cerrado sensu stricto; 3 ) $6 \mathrm{~km}$ road in the northwest edge of the reserve between cerrado sensu stricto and sugar cane monoculture; 4) $2 \mathrm{~km}$ road between cerrado sensu stricto and the reservoir, 5) $1.11 \mathrm{~km}$ road in the south-east edge of the reserve between cerradão and pastureland and 6) $1 \mathrm{~km}$ road that surrounds a small village. We often visited one or more vegetation forms during a single visit. We sampled cerradão on seven visits, cerrado sensu stricto on 22 , gallery forest on 10 , reservoir border on 14 and anthropic areas on 21 . 
We used $8 \times 40$ binoculars for bird identification, and pictures or vocalisation records for comparison and identification according to literature (Meyer de Schauensee, 1982; Sick, 1997; Souza, 1998; Develey and Endrigo, 2004) and to Manoel M. Dias' identification. We carried out a non-standardised method of observation, because our aim was to identify as many individuals as possible. Bird nomenclature follows Comitê Brasileiro de Registros Ornitológicos (2007). Bird species endemic to Cerrado were classified following Silva and Bates (2002), and migratory birds according to Willis (1979), Motta-Junior (1990) and Sick (1997). We identified threatened species from official lists [São Paulo, 2008; Instituto Brasileiro do Meio Ambiente e dos Recursos Naturais Renováveis (IBAMA), 2003; International Union for Conservation of Nature and Natural Resources (IUCN), 2007]. We measured the frequency of occurrence (FO) for each spe- cies in each vegetation form, because sample effort was different among them. We calculated FO dividing the total number of records of each species by the total number of visits to each area (Vielliard and Silva, 1990; Aleixo and Vielliard, 1995). Also, we classified species into nine trophic categories according to their principal feeding habits: nectarivores (NEC), carrion-eaters (CAE), carnivores (CAR), frugivores (FRU), granivores (GRA), omnivores (ONI), insectivores (INS), piscivores (PIS) and herbivores (HER), following Motta-Junior (1990), Sick (1997), and also our field observations.

\section{Results}

We found 160 bird species from 19 orders and 48 families (Table 1). Ninety-two (57.5\%) were Passeriformes and $68(42.5 \%)$ non-Passeriformes. We

Table 1. Bird species recorded in a cerrado fragment and surroundings at Fazenda Canchim, São Carlos, SP, from May 2004 to December 2006, and their respective frequency of occurrence (FO) in habitats (CA = cerradão; $\mathrm{CE}=$ cerrado sensu stricto; $\mathrm{GF}=$ gallery forest $\mathrm{RB}=$ reservoir border, $\mathrm{PS}=$ pastureland and sugar cane monoculture, $\mathrm{AA}=$ anthropic area), trophic categories $(\mathrm{TC})(\mathrm{NEC}=$ nectarivores, $\mathrm{CAE}=$ carrion-eaters, $\mathrm{CAR}=$ carnivores, $\mathrm{FRU}=$ frugivores, $\mathrm{GRA}=$ granivores, $\mathrm{ONI}=$ omnivores, INS $=$ insectivores, PIS $=$ piscivores and HER $=$ herbivores $).$ Blank spaces means FO values $=0$.

\begin{tabular}{|c|c|c|c|c|c|c|c|}
\hline \multirow[t]{2}{*}{ Taxon } & \multicolumn{6}{|c|}{ FO (\%) } & \multirow[t]{2}{*}{ TC } \\
\hline & CA & $\mathbf{C E}$ & GF & RB & PS & $\mathbf{A A}$ & \\
\hline \multicolumn{8}{|l|}{ Tinamiformes } \\
\hline \multicolumn{8}{|l|}{ TINAMIDAE } \\
\hline Crypturellus parvirostris (Wagler, 1827) & 14.3 & 68.2 & - & - & 18.2 & 14.3 & $\mathrm{ONI}^{1}$ \\
\hline \multicolumn{8}{|l|}{ Pelecaniformes } \\
\hline \multicolumn{8}{|l|}{ PHALACROCORACIDAE } \\
\hline Phalacrocorax brasilianus (Gmelin, 1789) & - & - & - & 50.0 & - & - & $\mathrm{PIS}^{2}$ \\
\hline \multicolumn{8}{|l|}{ Ciconiiformes } \\
\hline \multicolumn{8}{|l|}{ ARDEIDAE } \\
\hline Ardea cocoi Linnaeus, 1766 & - & - & - & 14.3 & - & - & $\mathrm{PIS}^{2}$ \\
\hline Ardea alba Linnaeus, 1758 & - & - & - & 100.0 & - & - & $\mathrm{PIS}^{2}$ \\
\hline Egretta thula (Molina, 1782) & - & - & - & 50.0 & - & - & $\mathrm{PIS}^{2}$ \\
\hline Bubulcus ibis (Linnaeus, 1758) & - & - & - & 21.4 & 40.9 & - & $\mathrm{INS}^{2}$ \\
\hline Butorides striatus (Linnaeus, 1758) & - & - & - & 50.0 & - & - & $\mathrm{PIS}^{2}$ \\
\hline Syrigma sibilatrix (Temminck, 1824) & - & - & - & - & 13.6 & - & INS $^{2}$ \\
\hline Nycticorax nycticorax (Linnaeus, 1758) & - & - & - & 21.4 & - & - & $\mathrm{PIS}^{2}$ \\
\hline Tigrisoma lineatum (Boddaert, 1783) & - & - & - & 7.1 & - & - & $\mathrm{PIS}^{2}$ \\
\hline \multicolumn{8}{|l|}{ THRESKIORNITHIDAE } \\
\hline Mesembrinibis cayennensis (Gmelin, 1789) & - & - & 20.0 & 78.6 & - & - & $\mathrm{INS}^{2}$ \\
\hline \multicolumn{8}{|l|}{ Cathartiformes } \\
\hline \multicolumn{8}{|l|}{ CATHARTIDAE } \\
\hline Coragyps atratus (Bechstein, 1793) & - & 18.2 & - & 7.1 & 27.3 & 14.3 & $\mathrm{CAE}^{1}$ \\
\hline \multicolumn{8}{|l|}{ Anseriformes } \\
\hline \multicolumn{8}{|l|}{ ANATIDAE } \\
\hline Dendrocygna viduata (Linnaeus, 1766) M & - & - & - & 7.1 & - & - & $\mathrm{HER}^{2}$ \\
\hline \multicolumn{8}{|l|}{ Falconiformes } \\
\hline \multicolumn{8}{|l|}{ ACCIPITRIDAE } \\
\hline Elanus leucurus (Vieillot, 1818) & - & 4.5 & - & - & 4.5 & - & $\mathrm{CAR}^{2}$ \\
\hline
\end{tabular}

${ }^{1}$ Motta-Junior (1990); ${ }^{2}$ Sick (1997); T = Threatened species; Status: V = vulnerable, CR = critically endangered, E = endangered, $\mathrm{R}=$ near threatened, $\mathrm{L}=$ least concern; ${ }^{\mathrm{S}}$ Threatened at the State of São Paulo; ${ }^{\mathrm{w}}$ Threatened in world; D = endemism in relation to Cerrado; $\mathrm{M}=$ migratory habit. 
Table 1. Continued...

\begin{tabular}{|c|c|c|c|c|c|c|c|}
\hline \multirow[t]{2}{*}{ Taxon } & \multicolumn{6}{|c|}{ FO $(\%)$} & \multirow[t]{2}{*}{ TC } \\
\hline & CA & $\mathbf{C E}$ & GF & $\mathbf{R B}$ & PS & $\mathbf{A A}$ & \\
\hline Ictinia plumbea (Gmelin, 1788) M & - & - & - & - & 4.5 & - & INS $^{1}$ \\
\hline Rupornis magnirostris (Gmelin, 1788) & - & 27.3 & - & - & 22.7 & 9.5 & $\mathrm{CAR}^{1}$ \\
\hline \multicolumn{8}{|l|}{ FALCONIDAE } \\
\hline Milvago chimachima (Vieillot, 1816) & - & 9.1 & - & - & 9.1 & - & CAR $^{1}$ \\
\hline Caracara plancus (Miller, 1777) & 14.3 & 27.3 & - & 7.1 & 22.7 & 9.5 & $\mathrm{CAR}^{1}$ \\
\hline Falco sparverius Linnaeus, 1758 & - & - & - & - & 4.5 & - & $\mathrm{INS}^{2}$ \\
\hline Falco femoralis Temminck, 1822 & - & 9.1 & - & - & 4.5 & - & CAR $^{1}$ \\
\hline \multicolumn{8}{|l|}{ Galiformes } \\
\hline \multicolumn{8}{|l|}{ CRACIDAE } \\
\hline Penelope superciliaris Temminck, 1815 & - & - & 10.0 & - & - & - & $\mathrm{FRU}^{2}$ \\
\hline \multicolumn{8}{|l|}{ Gruiformes } \\
\hline \multicolumn{8}{|l|}{ RALLIDAE } \\
\hline Aramides cajanea (Statius Muller, 1776) & - & - & - & 14.3 & - & - & $\mathrm{ONI}^{2}$ \\
\hline \multicolumn{8}{|l|}{ CARIAMIDAE } \\
\hline Cariama cristata (Linnaeus, 1766) & - & 13.6 & - & - & 54.5 & - & INS $^{1}$ \\
\hline \multicolumn{8}{|l|}{ Charadriiformes } \\
\hline \multicolumn{8}{|l|}{ JACANIDAE } \\
\hline Jacana jacana (Linnaeus, 1766) & - & - & - & 71.4 & - & - & $\mathrm{ONI}^{2}$ \\
\hline \multicolumn{8}{|l|}{ CHARADRIIDAE } \\
\hline Vanellus chilensis (Molina, 1782) & - & - & - & 28.6 & 54.5 & - & $\mathrm{ONI}^{2}$ \\
\hline \multicolumn{8}{|l|}{ RECURVIROSTRIDAE } \\
\hline Himantopus melanurus Vieillot, 1817 & - & - & - & 21.4 & - & - & CAR \\
\hline \multicolumn{8}{|l|}{ Columbiformes } \\
\hline \multicolumn{8}{|l|}{ COLUMBIDAE } \\
\hline Columbina talpacoti (Temminck, 1811) & - & 31.8 & - & - & - & 57.1 & GRA $^{1}$ \\
\hline Columbina squammata (Lesson, 1831) & - & 9.1 & - & - & - & 4.8 & $\mathrm{GRA}^{2}$ \\
\hline Patagioenas picazuro (Temminck, 1813) & 28.6 & 45.5 & 10.0 & - & 13.6 & 28.6 & FRU $^{1}$ \\
\hline Patagioneas cayennensis (Bonnaterre, 1792) & - & 31.8 & - & - & - & 4.8 & $\mathrm{FRU}^{2}$ \\
\hline Zenaida auriculata (Des Murs, 1847) & - & 9.1 & - & - & - & 28.6 & GRA $^{1}$ \\
\hline Leptotila verreauxi Bonaparte, 1855 & 14.3 & 45.5 & 30.0 & - & - & 4.8 & $\mathrm{FRU}^{1}$ \\
\hline Leptotila rufaxilla (Richard and Bernard, 1792) & 14.3 & - & - & - & - & 4.8 & $\mathrm{FRU}^{2}$ \\
\hline \multicolumn{8}{|l|}{ Psittaciformes } \\
\hline \multicolumn{8}{|l|}{ PSITTACIDAE } \\
\hline Aratinga leucophthalma (Statius Muller, 1776) & - & 22.7 & - & - & 13.6 & 14.3 & $\mathrm{FRU}^{2}$ \\
\hline Aratinga aurea (Gmelin, 1788) & - & 4.5 & - & - & - & 9.5 & $\mathrm{FRU}^{2}$ \\
\hline Forpus xanthopterygius (Spix, 1824) & - & 4.5 & - & - & - & - & $\mathrm{FRU}^{2}$ \\
\hline Brotogeris chiriri (Vieillot, 1818) & - & 45.5 & - & - & 18.2 & 23.8 & $\mathrm{FRU}^{1}$ \\
\hline Amazona aestiva (Linnaeus, 1758) & - & 9.1 & - & - & - & - & $\mathrm{FRU}^{2}$ \\
\hline \multicolumn{8}{|l|}{ Cuculiformes } \\
\hline \multicolumn{8}{|l|}{ CUCULIDAE } \\
\hline Piaya cayana (Linnaeus, 1766) & 14.3 & 18.2 & - & - & - & - & INS $^{1}$ \\
\hline Crotophaga ani Linnaeus, 1758 & - & 4.5 & - & - & - & 28.6 & INS $^{1}$ \\
\hline Guira guira (Gmelin, 1788) & - & 4.5 & - & - & - & 19.0 & INS $^{2}$ \\
\hline Tapera naevia (Linnaeus, 1766) & - & 27.3 & - & - & - & - & INS $^{1}$ \\
\hline \multicolumn{8}{|l|}{ Strigiformes } \\
\hline TYTONIDAE & & & & & & & \\
\hline
\end{tabular}

Tyto alba (Scopoli, 1769)

$9.5 \mathrm{CAR}^{2}$

${ }^{1}$ Motta-Junior (1990); ${ }^{2}$ Sick (1997); T = Threatened species; Status: V = vulnerable, CR = critically endangered, E = endangered, $\mathrm{R}=$ near threatened, $\mathrm{L}=$ least concern; ${ }^{\mathrm{S}}$ Threatened at the State of São Paulo; ${ }^{\mathrm{W}}$ Threatened in world; $\mathrm{D}=\mathrm{ende-}$ mism in relation to Cerrado; $\mathrm{M}=$ migratory habit. 
Table 1. Continued...

\begin{tabular}{|c|c|c|c|c|c|c|c|}
\hline \multirow[t]{2}{*}{ Taxon } & \multicolumn{6}{|c|}{ FO $(\%)$} & \multirow[t]{2}{*}{ TC } \\
\hline & CA & $\mathbf{C E}$ & GF & $\mathbf{R B}$ & PS & $\mathbf{A A}$ & \\
\hline \multicolumn{8}{|l|}{ STRIGIDAE } \\
\hline Megascops choliba (Vieillot, 1817) & - & 4.5 & - & - & - & - & $\mathrm{INS}^{2}$ \\
\hline Athene cunicularia (Molina, 1782) & - & - & - & - & 18.2 & - & $\mathrm{INS}^{2}$ \\
\hline Asio flammeus (Pontoppidan, 1763) T $\left(\mathbf{E}^{\mathrm{S}}\right)$ & - & - & - & - & 4.5 & - & $\mathrm{CAR}^{2}$ \\
\hline \multicolumn{8}{|l|}{ Caprimulgiformes } \\
\hline \multicolumn{8}{|l|}{ CAPRIMULGIDAE } \\
\hline Nyctidromus albicollis (Gmelin, 1789) & - & 22.7 & - & - & - & 19.0 & $\mathrm{INS}^{1}$ \\
\hline Caprimulgus rufus Boddaert, 1783 & - & 4.5 & - & - & - & - & $\mathrm{INS}^{2}$ \\
\hline \multicolumn{8}{|l|}{ Apodiformes } \\
\hline \multicolumn{8}{|l|}{ APODIDAE } \\
\hline Chaetura meridionalis Hellmayr, 1907 M & - & 4.5 & - & - & - & - & INS $^{2}$ \\
\hline \multicolumn{8}{|l|}{ TROCHILIDAE } \\
\hline Phaethornis pretrei (Lesson and Delattre, 1839) & - & 22.7 & - & - & - & - & $\mathrm{NEC}^{1}$ \\
\hline Eupetomena macroura (Gmelin, 1788) & - & 13.6 & - & - & - & 4.8 & $\mathrm{NEC}^{1}$ \\
\hline Colibri serrirostris (Vieillot, 1816) & - & 9.1 & - & - & - & - & $\mathrm{NEC}^{1}$ \\
\hline Chlorostilbon lucidus (d'Orbigny and Lafresnaye, 1838) & - & 27.3 & - & - & - & - & $\mathrm{NEC}^{1}$ \\
\hline Hylocharis chrysura (Shaw, 1812) $\mathbf{T}\left(\mathbf{L}^{\mathbf{w}}\right)$ & - & 9.1 & - & - & - & - & $\mathrm{NEC}^{1}$ \\
\hline Amazilia lactea (Lesson, 1832) & - & 22.7 & - & - & - & - & $\mathrm{NEC}^{1}$ \\
\hline Heliomaster squamosus (Temminck, 1823) & - & 4.5 & - & - & - & - & $\mathrm{NEC}^{2}$ \\
\hline \multicolumn{8}{|l|}{ Coraciiformes } \\
\hline \multicolumn{8}{|l|}{ ALCEDINIDAE } \\
\hline Ceryle torquatus (Linnaeus, 1766) & - & - & - & 42.9 & - & - & $\mathrm{PIS}^{2}$ \\
\hline Chloroceryle amazona (Latham, 1790) & - & - & - & 28.6 & - & - & $\mathrm{PIS}^{2}$ \\
\hline Chloroceryle americana (Gmelin, 1788) & - & - & - & 14.3 & - & - & $\mathrm{PIS}^{2}$ \\
\hline \multicolumn{8}{|l|}{ MOMOTIDAE } \\
\hline Baryphthengus ruficapillus (Vieillot, 1818) & - & 9.1 & - & - & - & - & $\mathrm{INS}^{2}$ \\
\hline \multicolumn{8}{|l|}{ Galbuliformes } \\
\hline \multicolumn{8}{|l|}{ GALBULIDAE } \\
\hline Galbula ruficauda Cuvier, 1816 & - & 9.1 & - & - & - & - & $\mathrm{INS}^{2}$ \\
\hline \multicolumn{8}{|l|}{ Piciformes } \\
\hline \multicolumn{8}{|l|}{ RAMPHASTIDAE } \\
\hline Ramphastos toco Statius Mullier, 1776 & - & 4.5 & - & - & - & - & $\mathrm{FRU}^{2}$ \\
\hline \multicolumn{8}{|l|}{ PICIDAE } \\
\hline Picumnus albosquamatus d'Orbigny, 1840 & 28.6 & 22.7 & 20.0 & - & - & - & $\mathrm{INS}^{2}$ \\
\hline Colaptes campestris (Vieillot, 1818) & - & 40.9 & - & - & 18.2 & 19.0 & INS $^{1}$ \\
\hline Colaptes melanochloros (Gmelin, 1788) & - & 40.9 & - & - & - & - & INS $^{1}$ \\
\hline Dryocopus lineatus (Linnaeus, 1766) & 57.1 & 27.3 & - & - & - & 9.5 & INS $^{1}$ \\
\hline Melanerpes candidus (Otto, 1796) & - & 13.6 & - & - & - & - & INS $^{1}$ \\
\hline Veniliornis passerinus (Linnaeus, 1766) & - & 4.5 & - & - & - & - & INS $^{1}$ \\
\hline \multicolumn{8}{|l|}{ Passeriformes } \\
\hline \multicolumn{8}{|l|}{ MELANOPAREIIDAE } \\
\hline Melanopareia torquata (Wied, 1831) D, T (E) & - & 4.5 & - & - & - & - & INS $^{1}$ \\
\hline \multicolumn{8}{|l|}{ THAMNOPHILIDAE } \\
\hline Taraba major (Vieillot, 1816) & - & 9.1 & - & - & - & - & INS $^{1}$ \\
\hline Thamnophilus doliatus (Linnaeus, 1764) & - & 59.1 & - & - & - & 28.6 & $\mathrm{INS}^{2}$ \\
\hline Thamnophilus pelzelni Hellmayr, 1924 & 14.3 & 54.5 & - & - & - & - & INS $^{1}$ \\
\hline
\end{tabular}

${ }^{1}$ Motta-Junior (1990); ${ }^{2}$ Sick (1997); T = Threatened species; Status: V = vulnerable, CR = critically endangered, E = endangered, $\mathrm{R}=$ near threatened, $\mathrm{L}=$ least concern; ${ }^{\mathrm{S}}$ Threatened at the State of São Paulo; ${ }^{\mathrm{W}}$ Threatened in world; $\mathrm{D}=$ endemism in relation to Cerrado; $\mathrm{M}=$ migratory habit. 
Table 1. Continued...

\begin{tabular}{|c|c|c|c|c|c|c|c|}
\hline \multirow[t]{2}{*}{ Taxon } & \multicolumn{6}{|c|}{ FO $(\%)$} & \multirow[t]{2}{*}{ TC } \\
\hline & CA & $\mathbf{C E}$ & GF & $\mathbf{R B}$ & PS & $\mathbf{A A}$ & \\
\hline Thamnophilus caerulescens Vieillot, 1816 & - & 31.8 & - & - & - & - & $\mathrm{INS}^{1}$ \\
\hline Formicivora rufa (Wied, 1831) & 14.3 & 4.5 & - & - & - & - & $\mathrm{INS}^{1}$ \\
\hline Herpsilochmus atricapillus Pelzeln, 1868 & - & 9.1 & - & - & - & - & $\mathrm{INS}^{2}$ \\
\hline \multicolumn{8}{|l|}{ FURNARIIDAE } \\
\hline Furnarius rufus (Gmelin, 1788) & - & 18.2 & - & - & - & 76.2 & $\mathrm{INS}^{2}$ \\
\hline Synallaxis spixi Sclater, 1856 & - & 36.4 & - & - & - & - & INS $^{1}$ \\
\hline Synallaxis frontalis Pelzeln, 1859 & - & 45.5 & - & - & - & - & INS $^{1}$ \\
\hline Certhiaxis cinnamomeus (Gmelin, 1788) & - & 22.7 & - & - & - & - & $\mathrm{INS}^{2}$ \\
\hline Automolus leucophthalmus (Wied, 1821) & - & 4.5 & - & - & - & - & $\mathrm{INS}^{1}$ \\
\hline Xenops rutilans Temminck, 1821 & - & - & 10.0 & - & - & - & INS $^{1}$ \\
\hline \multicolumn{8}{|l|}{ DENDROCOLAPTIDAE } \\
\hline Lepidocolaptes angustirostris (Vieillot, 1818) & - & 36.4 & - & - & - & 9.5 & INS $^{1}$ \\
\hline \multicolumn{8}{|l|}{ TYRANNIDAE } \\
\hline Camptostoma obsoletum (Temminck, 1824) & 28.6 & 45.5 & - & - & - & 9.5 & INS $^{1}$ \\
\hline Phaeomyias murina (Spix, 1825) & - & 13.6 & - & - & - & - & $\mathrm{INS}^{2}$ \\
\hline Suiriri suiriri (Vieillot, 1818) $\mathrm{T}\left(\mathrm{CR}^{\mathrm{S}}\right)$ & - & 4.5 & - & - & - & - & INS $^{1}$ \\
\hline Elaenia flavogaster (Thunberg, 1822) & - & 22.7 & - & - & - & 66.7 & $\mathrm{ONI}^{1}$ \\
\hline Elaenia mesoleuca (Deppe, 1830) M & - & 4.5 & - & - & - & - & $\mathrm{ONI}^{1}$ \\
\hline Elaenia chiriquensis Lawrence, 1865 M & - & 22.7 & - & - & - & - & $\mathrm{ONI}^{1}$ \\
\hline Elaenia obscura (d'Orbigny and Lafresnaye, 1837) & - & 4.5 & - & - & - & - & $\mathrm{ONI}^{1}$ \\
\hline Euscarthmus meloryphus Wied, 1831 & - & 18.2 & - & - & - & - & $\mathrm{INS}^{2}$ \\
\hline $\begin{array}{l}\text { Hemitriccus margaritaceiventer } \\
\text { (d'Orbigny and afresnaye, 1827) }\end{array}$ & - & 9.1 & - & - & - & - & INS $^{1}$ \\
\hline Todirostrum poliocephalum (Wied, 1831) & - & 4.5 & - & - & - & - & $\mathrm{INS}^{2}$ \\
\hline Tolmomyias sulphurescens (Spix, 1825) & 14.3 & - & - & - & - & - & INS $^{1}$ \\
\hline Hirundinea ferruginea (Gmelin, 1788) M & - & - & - & - & - & 9.5 & $\mathrm{INS}^{2}$ \\
\hline Lathrotriccus euleri (Cabanis, 1868) & - & 18.2 & - & - & - & - & $\mathrm{INS}^{2}$ \\
\hline Cnemotriccus fuscatus (Wied, 1831) & - & 13.6 & - & - & - & - & $\mathrm{INS}^{2}$ \\
\hline Pyrocephalus rubinus (Boddaert, 1783) M & - & 4.5 & - & - & - & - & INS $^{1}$ \\
\hline Xolmis velatus (Lichstenstein, 1823) M & - & - & - & - & 9.1 & 9.5 & $\mathrm{INS}^{2}$ \\
\hline Fluvicola nengeta (Linnaeus, 1766) & - & - & - & 14.3 & - & - & $\mathrm{INS}^{2}$ \\
\hline Arundinicola leucocephala (Linnaeus, 1764) & - & - & - & 42.9 & - & - & $\mathrm{INS}^{2}$ \\
\hline Casiornis rufus (Vieillot, 1816) M & - & 4.5 & - & - & - & - & INS $^{1}$ \\
\hline Myiarchus swainsoni Cabanis and Heine, 1859 M & - & 4.5 & - & - & - & - & INS $^{1}$ \\
\hline Myiarchus ferox (Gmelin, 1789) & 14.3 & 4.5 & - & - & - & - & INS $^{1}$ \\
\hline Myiarchus tyrannulus (Statius Muller, 1766) & - & 18.2 & - & - & - & - & INS $^{1}$ \\
\hline Pitangus sulphuratus (Linnaeus, 1766) & - & 22.7 & - & - & - & 76.2 & $\mathrm{ONI}^{1}$ \\
\hline Megarhynchus pitangua (Linnaeus, 1766) & 14.3 & 36.4 & - & - & - & 33.3 & $\mathrm{ONI}^{1}$ \\
\hline Myiozetetes similis (Spix, 1825) & - & - & 30.0 & 64.3 & - & - & $\mathrm{ONI}^{1}$ \\
\hline Myiodynastes maculatus (Statius Muller, 1766) M & 28.6 & 31.8 & - & - & - & - & $\mathrm{ONI}^{1}$ \\
\hline Empidonomus varius (Vieillot, 1818) M & - & 22.7 & - & - & - & - & INS $^{1}$ \\
\hline Tyrannus savana Vieillot, $1808 \mathbf{M}$ & - & 27.3 & - & - & 13.6 & 9.5 & INS $^{1}$ \\
\hline Tyrannus melancholicus Vieillot, 1819 M & 14.3 & 31.8 & - & - & - & 61.9 & INS $^{1}$ \\
\hline \multicolumn{8}{|l|}{ PIPRIDAE } \\
\hline Antilophia galeata (Lichtenstein, 1823) D & - & - & 90.0 & - & - & - & FRU \\
\hline TITYRIDAE & & & & & & & \\
\hline
\end{tabular}

${ }^{1}$ Motta-Junior (1990); ${ }^{2}$ Sick (1997); T = Threatened species; Status: V = vulnerable, CR = critically endangered, E = endangered, $\mathrm{R}=$ near threatened, $\mathrm{L}=$ least concern; ${ }^{\mathrm{S}}$ Threatened at the State of São Paulo; ${ }^{\mathrm{W}}$ Threatened in world; $\mathrm{D}=$ endemism in relation to Cerrado; $\mathrm{M}=$ migratory habit. 
Table 1. Continued...

\begin{tabular}{|c|c|c|c|c|c|c|c|}
\hline \multirow[t]{2}{*}{ Taxon } & \multicolumn{6}{|c|}{ FO $(\%)$} & \multirow[t]{2}{*}{$\mathbf{T C}$} \\
\hline & CA & $\mathbf{C E}$ & GF & $\mathbf{R B}$ & PS & $\mathbf{A A}$ & \\
\hline Pachyramphus polychopterus (Vieillot, 1818) M & - & 4.5 & - & - & - & - & $\mathrm{INS}^{2}$ \\
\hline Pachyramphus validus (Lichtenstein, 1823) M & - & 9.1 & - & - & - & - & $\mathrm{INS}^{2}$ \\
\hline \multicolumn{8}{|l|}{ HIRUNDINIDAE } \\
\hline Tachycineta leucorrhoa (Vieillot, 1817) & - & - & - & - & - & 9.5 & INS $^{2}$ \\
\hline Pygochelidon cyanoleuca (Vieillot, 1817) & - & 22.7 & - & - & - & 14.3 & INS $^{2}$ \\
\hline Stelgidopteryx ruficollis (Vieillot, 1817) & - & 13.6 & - & - & - & 4.8 & $\mathrm{INS}^{2}$ \\
\hline \multicolumn{8}{|l|}{ CORVIDAE } \\
\hline Cyanocorax cristatellus (Temminck, 1823) D & - & 31.8 & - & - & - & 9.5 & $\mathrm{ONI}^{1}$ \\
\hline Cyanocorax chrysops (Vieillot, 1818) & 14.3 & 77.3 & - & - & - & 9.5 & $\mathrm{ONI}^{1}$ \\
\hline \multicolumn{8}{|l|}{ DONACOBIIDAE } \\
\hline Donacobius atricapilla (Linnaeus, 1766) & - & - & - & 7.1 & - & - & $\mathrm{INS}^{2}$ \\
\hline \multicolumn{8}{|l|}{ TROGLODYTIDAE } \\
\hline Cantorchilus leucotis (Lafresnaye, 1845) & - & 13.6 & 100.0 & - & - & 4.8 & $\mathrm{INS}^{1}$ \\
\hline Troglodytes musculus Naumann, 1823 & - & 36.4 & - & - & - & 19.0 & INS $^{1}$ \\
\hline \multicolumn{8}{|l|}{ TURDIDAE } \\
\hline Turdus rufiventris Vieillot, 1818 & 14.3 & - & - & - & - & 23.8 & $\mathrm{ONI}^{1}$ \\
\hline Turdus leucomelas Vieillot, 1818 & 14.3 & 13.6 & - & - & - & 61.9 & $\mathrm{ONI}^{1}$ \\
\hline Turdus amaurochalinus Cabanis, 1850 & - & 13.6 & - & - & - & 38.1 & $\mathrm{ONI}^{1}$ \\
\hline \multicolumn{8}{|l|}{ MIMIDAE } \\
\hline Mimus saturninus (Lichtenstein, 1823) & - & 22.7 & - & - & 18.2 & 33.3 & $\mathrm{ONI}^{2}$ \\
\hline \multicolumn{8}{|l|}{ VIREONIDAE } \\
\hline Cyclarhis gujanensis (Gmelin, 1789) & - & 27.3 & - & - & - & 9.5 & $\mathrm{INS}^{1}$ \\
\hline Vireo olivaceus (Linnaeus, 1766) M & 14.3 & 27.3 & - & - & - & 14.3 & $\mathrm{ONI}^{1}$ \\
\hline \multicolumn{8}{|l|}{ PARULIDAE } \\
\hline Parula pitiayumi (Vieillot, 1817) & - & 9.1 & - & - & - & - & INS $^{1}$ \\
\hline Geothlypis aequinoctialis (Gmelin, 1789) & - & 22.7 & - & 14.3 & - & - & $\mathrm{INS}^{2}$ \\
\hline Basileuterus flaveolus (Baird, 1865) & 14.3 & 36.4 & 60.0 & - & - & 9.5 & INS $^{1}$ \\
\hline Basileuterus leucophrys Pelzeln, $1868 \mathbf{D}, \mathbf{T}\left(\mathbf{E}^{\mathbf{S}}\right)$ & - & - & 20.0 & - & - & - & INS $^{1}$ \\
\hline Basileuterus hypoleucus Bonaparte, 1830 & 14.3 & 36.4 & 30.0 & - & - & 4.8 & $\mathrm{INS}^{1}$ \\
\hline \multicolumn{8}{|l|}{ COEREBIDAE } \\
\hline Coereba flaveola (Linnaeus, 1758) & 14.3 & 9.1 & - & - & - & 4.8 & $\mathrm{NEC}^{1}$ \\
\hline \multicolumn{8}{|l|}{ THRAUPIDAE } \\
\hline Schistochlamys ruficapillus (Vieillot, 1817) & 28.6 & 18.2 & - & - & - & - & $\mathrm{ONI}^{1}$ \\
\hline Nemosia pileata (Boddaert, 1783) & - & 18.2 & - & - & - & - & $\mathrm{INS}^{1}$ \\
\hline Tachyphonus coronatus (Vieillot, 1822) & - & 4.5 & - & - & - & - & $\mathrm{ONI}^{1}$ \\
\hline Ramphocelus carbo (Pallas, 1764) & - & 18.2 & - & - & - & - & $\mathrm{ONI}^{1}$ \\
\hline Thraupis sayaca (Linnaeus, 1766) & 14.3 & 50.0 & - & - & - & 38.1 & $\mathrm{ONI}^{1}$ \\
\hline Tangara cayana (Linnaeus, 1766) & 14.3 & 31.8 & - & - & - & 9.5 & $\mathrm{ONI}^{1}$ \\
\hline Tersina viridis (Illiger, 1811) M & - & 9.1 & - & - & - & - & $\mathrm{ONI}^{1}$ \\
\hline Dacnis cayana (Linnaeus, 1766) & 14.3 & 22.7 & - & - & - & 4.8 & $\mathrm{ONI}^{1}$ \\
\hline Conirostrum speciosum (Temminck, 1824) & 28.6 & 27.3 & - & - & - & - & $\mathrm{INS}^{2}$ \\
\hline \multicolumn{8}{|l|}{ EMBEREZIDAE } \\
\hline Zonotrichia capensis (Statius Muller, 1776) & - & 22.7 & - & - & 27.3 & 52.4 & GRA $^{1}$ \\
\hline Ammodramus humeralis (Bosc, 1792) & - & - & - & - & 45.5 & - & $\mathrm{GRA}^{2}$ \\
\hline Sicalis flaveola (Linnaeus, 1766) & - & - & - & - & 4.5 & - & $\mathrm{GRA}^{2}$ \\
\hline Sicalis luteola (Sparrman, 1789) M & - & - & - & 14.3 & - & - & $\mathrm{GRA}^{2}$ \\
\hline
\end{tabular}


Table 1. Continued...

\begin{tabular}{|c|c|c|c|c|c|c|c|}
\hline \multirow[t]{2}{*}{ Taxon } & \multicolumn{6}{|c|}{ FO $(\%)$} & \multirow[t]{2}{*}{ TC } \\
\hline & CA & $\mathbf{C E}$ & GF & $\mathbf{R B}$ & PS & $\mathbf{A A}$ & \\
\hline Volatinia jacarina (Linnaeus, 1766) & - & - & - & - & 36.4 & - & GRA $^{1}$ \\
\hline Sporophila collaris (Boddaert, 1783) $\mathbf{M}, \mathbf{T}\left(\mathbf{V}^{\mathbf{s}}\right.$ ) & - & 9.1 & - & - & - & - & $\mathrm{GRA}^{2}$ \\
\hline Sporophila lineola (Linnaeus, 1758) M & - & - & - & 21.4 & - & - & $\mathrm{GRA}^{2}$ \\
\hline Sporophila caerulescens (Vieillot, 1823) & - & 13.6 & - & - & 50.0 & - & $\mathrm{GRA}^{1}$ \\
\hline Arremon flavirostris Swainson, 1838 & - & - & 20.0 & - & - & - & INS $^{1}$ \\
\hline Charitospiza eucosma Oberholser $1905 \mathrm{D}, \mathrm{T}\left(\mathrm{CR}^{\mathrm{S}}, \mathrm{R}^{\mathrm{W}}\right)$ & - & 28.6 & - & - & - & - & $\mathrm{GRA}^{2}$ \\
\hline Coryphospingus cucullatus (Statius Muller, 1766) & - & 68.2 & - & - & 9.1 & 14.3 & $\mathrm{GRA}^{1}$ \\
\hline \multicolumn{8}{|l|}{ CARDINALIDAE } \\
\hline Saltator similis d'Orbigny and Lafresnaye, 1837 & - & 9.1 & - & - & - & 4.8 & $\mathrm{ONI}^{2}$ \\
\hline Saltator atricollis Vieillot, $1817 \mathbf{D}, \mathbf{T}\left(\mathbf{V}^{\mathbf{S}}\right)$ & - & - & - & - & - & - & $\mathrm{ONI}^{2}$ \\
\hline \multicolumn{8}{|l|}{ ICTERIDAE } \\
\hline Chrysomus ruficapillus (Vieillot, 1819) M & - & - & - & 57.1 & - & - & $\mathrm{ONI}^{2}$ \\
\hline Molothrus bonariensis (Gmelin, 1789) & - & - & - & - & - & 9.5 & $\mathrm{ONI}^{2}$ \\
\hline \multicolumn{8}{|l|}{ FRINGILLIDAE } \\
\hline Carduelis magellanica (Vieillot, 1805) & - & - & - & - & - & 4.8 & $\mathrm{GRA}^{2}$ \\
\hline Euphonia chlorotica (Linnaeus, 1766) & 14.3 & 18.2 & - & - & - & 9.5 & $\mathrm{ONI}^{1}$ \\
\hline
\end{tabular}

${ }^{1}$ Motta-Junior (1990); ${ }^{2}$ Sick (1997); T = Threatened species; Status: V = vulnerable, CR = critically endangered, E = endangered, $\mathrm{R}=$ near threatened, $\mathrm{L}=$ least concern; ${ }^{\mathrm{S}}$ Threatened at the State of São Paulo; ${ }^{\text {w }}$ Threatened in world; D = endemism in relation to Cerrado; $\mathrm{M}=$ migratory habit.

Table 2. Frequency of occurrence (\%) of bird species in each habitat at RL-CPPSE and surroundings.

\begin{tabular}{lcccc}
\hline \multirow{2}{*}{ Habitat } & \multicolumn{3}{c}{ FO } \\
\cline { 2 - 5 } & Up to 25 & $\mathbf{2 5 . 1 - 5 0}$ & $\mathbf{5 0 . 1 - 7 5}$ & $\mathbf{7 5 . 1 - 1 0 0}$ \\
\hline Cerradão & 95.6 & 3.8 & 0.6 & 0.0 \\
Cerrado sensu stricto & 78.1 & 18.8 & 2.5 & 0.6 \\
Gallery forest & 96.3 & 1.9 & 0.6 & 1.3 \\
Reservoir border & 92.5 & 4.4 & 1.9 & 1.3 \\
Pastureland and sugar cane monoculture & 95.0 & 3.8 & 1.3 & 0.0 \\
Anthropic area & 90.6 & 5.0 & 3.1 & 1.3 \\
\hline
\end{tabular}

found six species endemic to the Cerrado (Melanopareia torquata, Antilophia galeata, Cyanocorax cristatellus, Basileuterus leucophrys, Charitospiza eucosma and Saltator atricollis) and 22 migratory species (Table 1). Seven species were threatened in the State of São Paulo (Table 1): Suiriri suiriri and C. eucosma - critically endangered; Asio flammeus, $M$ torquata and B. leucophrys - endangered; S. atricollis and Sporophila collaris - vulnerable (São Paulo, 2008). Although we did not see nationally threatened species, we recorded two globally threatened (Table 1): Hylocharis chrysura - least concern and C. eucosma - near threatened (IUCN, 2007).

We found 28 species in the cerradão, 110 in the cerrado sensu stricto, 13 in the gallery forest, 26 in the
Table 3. Number of species (\% in parentheses) in trophic categories at RL-CPPSE and surroundings.

\begin{tabular}{cc}
\hline Trophic category & Number of species (\%) \\
\hline Insectivores & $75(46.9)$ \\
Omnivores & $31(19.4)$ \\
Granivores & $14(8.8)$ \\
Frugivores & $12(7.5)$ \\
Piscivores & $10(6.2)$ \\
Nectarivores & $8(5.0)$ \\
Carnivores & $8(5.0)$ \\
Carrion-eaters & $1(6)$ \\
Herbivores & $1(6)$ \\
Total & 160 \\
\hline
\end{tabular}


reservoir border, 26 in the pasturelands and sugar cane monoculture and 55 in the anthropic area (Table 1).

Most of the species had low FO values in all vegetation forms. In five of the six physiognomies, more than $90 \%$ of species were registered in less than $25 \%$ of the visits. Cerrado sensu stricto was the only one that presented almost $20 \%$ of bird species registered in 25.1 to $50 \%$ of the visits (Table 2). Insectivores were the major trophic category, followed by omnivores, granivores, piscivores, frugivores, carnivores, nectarivores, carrioneaters and herbivores (Table 3).

\section{Discussion}

\subsection{Species richness}

We found $20.8 \%$ of São Paulo avifauna (770 species) (Willis and Oniki, 2003) and $19.1 \%$ of Cerrado domain (Silva, 1995). Our results denote that RL-CPPSE has representative species richness if compared with other studies, even though there are few bird studies in São Paulo's cerrado fragments. Twenty years ago, in 4 hours of observation, Willis and Oniki (2003) found 89 species at Fazenda Canchim, in cerrado fragment and semideciduous forest. Other previous studies found 214 bird species in the campus of the Universidade Federal de São Carlos in 9 years of study (Motta-Junior and Vasconcellos, 1996), 302 in Estações Ecológicas de Jataí e Experimental de Luis Antônio in 13 years (Dias, 2000), 231 in Estação Ecológica de Itirapina in 21 years (Willis, 2004) and in 9 years (Motta-Junior et al., 2008), 144 species in Parque Estadual de Vassununga in 1 year (Develey et al., 2005) and 158 and 201 species in two fragments in Estação Experimental de Itirapina in 15 months (Telles and Dias, 2010). In addition, it is important to notice that RL-CPPSE is one of the larger remnants in the region (Primavesi et al., 1999) and could be suitable as a natural corridor or habitat and food source for birds.

\subsection{Endemic and threatened species}

We found $20 \%$ (six species) of all endemic species to Cerrado (Silva and Bates 2002); four of them were threatened in São Paulo (São Paulo, 2008). We registered Charitospiza eucosma, which was also recorded at Estação Ecológica de Itirapina, SP (Motta-Junior et al., 2008). Even though this species has been considered as missing or critically endangered in the state (Willis, 2004; São Paulo, 2008), these recent records provide evidence that there are populations breeding within São Paulo. The few Charitospiza eucosma records have been related to habitat loss, since it usually occurs in natural open areas subjected to regular fires (e.g., open savannas) (Willis, 2004), which are now rarely found in the state (Durigan et al., 1987; 2003). Furthermore, this species is considered high sensitive to habitat disturbance (Stotz et al., 1996). However, the lack of studies in cerrado fragments in the state may also contribute to C. eucosma threaten status. We suggest, then, more censuses and population monitoring should be conducted in these areas.
Among other endemic and threatened species in the state, we found Melanopareia torquata, which has been rarely studied (e.g. Tubelis and Cavalcanti, 2000). Recently, some population studies have been providing information about their breeding biology and ecology (Gressler and Marini, 2008; Kanegae et al., 2008). Although $M$. torquata is considered as medium-sensitive species to anthropic disturbance (Stotz et al., 1996), we suggest that it should receive high protection effort in São Paulo. Like M. torquata, A. flammeus is classified as endangered species in São Paulo (São Paulo, 2008). We registered this species only once, flying above pastureland with typical foraging.

\subsection{Frequency of occurrence}

We found low frequency of occurrence (FO) value for most species, which was also reported in other studies in fragments in São Paulo (Aleixo and Vielliard, 1995; Almeida et al., 1999; Donatelli et al. 2007). Few records for species might occur because of the unusual exploitation of the area, for instance feeding habits in the area during a short period of time. Alternatively, migratory and occasional species (see above) or species not easy to detect, such as hummingbirds that are difficult to see or hear, could have also contributed to FO reduced values. We were unable to compare FO between vegetation forms, because the number of visits in each area was not similar.

Migrants that were found indicate this site as an important shelter and food source. We observed both short- and long-distance migratory species, such as Dendrocygna viduata and Tyrannus savana, respectively. However, we did not observe three migratory species, Amazonetta brasiliensis (Gmelin, 1789), Platalea ajaja Linnaeus, 1758 and Rynchops niger Linnaeus, 1758, which used to be observed in the region (Manoel M. Dias, pers. comm.). This may be caused by non-annual site use, to area isolation or to habitat quality decrease in the reserve in recent years. However, our irregular sampling method could also have failed to register more migrant species.

\subsection{Bird species and vegetation forms}

Inside the protected area of cerrado, we found 120 species, $75 \%$ of the total recorded. In the cerradão, we registered bird species with canopy foraging habits, such as Dryocopus lineatus, Conirostrum speciosum and Schistochlamys ruficapillus (Stotz et al., 1996). These species had also the highest FO at this habitat. In the cerrado sensu stricto, we found typical species of savanna or similar vegetation forms, e.g. Colaptes campestres, which uses terrestrial and canopy foraging strata, and Melanopareia torquata, with terrestrial and understory habits (Stotz et al., 1996; Sick, 1997). Species with the high FO in this physiognomy were Cyanocorax chrysops, Crypturellus parvirostris, Coryphospingus cuculatus, Thamnophilus doliatus and T. punctatus. In the gallery forest, Cantorchilus leucotis, Antilophia galeata and 
Basileuterus flaveolus were the most frequent species. Despite the small area of gallery forest, this vegetation type usually shows high biodiversity. Some birds might use different strata (e.g. B. flaveolus, B. leucophrys and B. hypoleucus, Marini and Cavalcanti, 1993) allowing an increase in species richness (Macedo, 2002).

We found 92 species in the surrounding area, including pasturelands, sugar cane monoculture, reservoir border and anthropic area. Ammodramus humeralis, Sicalis flaveola, S. luteola, Sporophila spp. and Volatinia jacarina were typical grassland species found in pastureland borders. Among non-Passeriformes species, Cariama cristata, Syrigma sibilatrix and Bubulcus ibis were frequently found in pasturelands. They are usually benefited by agriculture and pastureland occupation (Sick, 1997). For instance, Bubulcus ibis, was first registered in Brazil almost 40 years ago, and rapidly became abundant (Sick, 1997). Individuals of this species usually follow grazing cattle, improving their ability to find insects for feeding (Sick, 1997).

Swampy species were registered at the reservoir border, such as Certhiaxis cinnamomea, Chrysomus ruficapillus, Fluvicola nengeta and Himantopus melanura, which forage on wet ground. Aquatic species, which are highy dependent of water for fish, aquatic insects or mollusks food items availability, were commonly registered in the area. Ardea alba had the highest FO in this habitat $(100 \%)$, followed by Mesembrinibis cayennensis $(78.6 \%)$ and Jacana jacana $(71.4 \%)$. We found a juvenile individual of Tigrisoma lineatum only once in the reservoir border, which indicates reproduction in the area.

In the anthropic area, including a small village, we frequently found many generalist species, like Pitangus sulphuratus and Furnarius rufus. Most Columbidae species are also highly adapted to anthropic areas and are easily found in these areas. Patagioenas picazuro, $P$. cayennensis and Zenaida auriculata were uncommon in São Paulo until the 1960's, but since then, they have become abundant in the state (Willis and Oniki 1987). The expansion of mechanical agriculture increases grain availability on the ground and therefore benefits these species' feeding behaviour (Willis and Oniki 1987)

\subsection{Trophic categories}

Species distribution in trophic categories follows similar results found in previous studies (Willis, 1979; Motta-Junior and Vasconcellos, 1996; Krügel and Anjos, 2000; Anjos, 2001; Tellino-Junior et al., 2005). Most species were insectivorous (46.9\%), a typical pattern in tropical regions (Sick, 1997; Macedo, 2002), probably related to site resources availability. Omnivorous species followed in the rank (19.4\%) and were also significant for the community composition. These species are expected to be less prejudiced in disturbed areas, because they have diverse diets (Willis, 1979; Motta-Junior, 1990).

Granivores were in third position in the categories rank. Such species records are probably related to pas- turelands and crop fields in the surrounding area that provide suitable foraging sites. Frugivores were fewer in number of species in our sample. However, we found small (Antilophia galeata), medium (Amazona aestiva) and large frugivores (Penelope superciliares and Ramphastos toco), which indicates the wide range of food item availability. This group is of great importance in natural area conservation, since it is strongly associated with seed dispersal and vegetation structure maintenance (Pizo, 2001).

Nectarivores were poorly represented in the bird community, and were composed almost entirely of hummingbird species. Most of these species are mutually related to some plant species, as well as frugivores, since they are pollinators. Seed production or germination of many plants species may be affected by the reduction of pollinators in fragmented areas (Murcia, 1996), which denotes the ecological importance of nectarivores. The category of Piscivores was mainly composed of Ardeidae species, which require aquatic environments for enough food availability (Sick, 1997). Therefore, on the RL-CPPSE boundary, Represa do 29 is a representative habitat for this group maintenance. Carnivorous species, such as hawks (Rupornis magnirostris) and owls (Tyto alba) registered in this study, are also critical for community dynamics, because they are top predators (Sick, 1997) and may control the population size of small animals.

\section{Conclusion}

We found remarkable data at RL-CPPSE, such as endemic and critically threatened bird species, and high species richness. Species distribution in trophic categories also indicates the area as a suitable environment for the existence of a wide range of birds. Therefore, here we contribute with valuable information for the development of conservation or management plans at a local or regional level. Regarding our results and the area features, such as its size, conservation status and position within the state - between Cerrado and Atlantic Forest - the study site must be considered as a priority conservation area to preserve bird species in the State of São Paulo.

Acknowledgements - We are grateful to FOG Figueiredo, KD Espartosa, MV Cianciaruso, MH Santos, RB Negrão and GC Molina for helping us in the field work. We are grateful to O. Primavesi and Embrapa Pecuária Sudeste, for allowing us to conduct this study in RL-CPPSE. We are grateful to MV Cianciaruso, L. Bezerra, MR Francisco and O. Rocha, for the comments and suggestions, and for two anonymous reviewers of the manuscript. LTM is grateful to PIBIC/CNPq, for the scholarship.

\section{References}

ALEIXO, A. and VIELLIARD, JME., 1995. Composição da avifauna da Mata de Santa Genebra,Campinas, São Paulo, Brasil. Revista Brasileira de Zoologia, vol. 12, no. 3, p. 493-511. 
ALHO, CJR. and MARTINS, ES., 1995. De grão em grão o cerrado perde espaço. Brasília: WWF.

ALMEIDA, MEC., VIELLIARD, JME. and DIAS, MM., 1999. Composição da avifauna em duasmatas ciliares do rio Jacaré-Pepira, São Paulo, Brasil. Revista Brasileira de Zoologia, vol. 16, no. 4, p. 1087-1098.

ANDRÉN, H., 1994. Effects of habitat fragmentation on birds and mammals in landscapes with different proportions of suitable habitat: a review. Oikos, vol. 71, no. 3, p. 355-366.

ANJOS, L., 2001. Bird communities in five Atlantic Forest fragments in Southern Brazil. Ornitologia Neotropical, vol. 12, no.1, p. 11-27.

BirdLife International, 2003. World Bird Database: the site for bird conservation. Version 2.0. Cambridge: BirdLife International. Available from: <http://www.birdlife.org $>$. Access in: 13/07/2008.

CAVALCANTI, RB. and JOLY, CA., 2002. Biodiversity and conservation priorities in the Cerrado region. In Oliveira, PS. and Marquis RJ. (Eds.). The cerrados of Brazil: ecology and natural history of a neotropical savanna. New York: Columbia University Press. p. 351-367.

Comitê Brasileiro de Registros Ornitológicos - CBRO, 2007. Listas das aves do Brasil. CBRO. Available from: <http://www. cbro.org.br>. Access in: 13/07/2008

DEBINSKI, DM. and HOLT, RD., 2000. A survey and overview of habitat fragmentation experiments. Conservation Biology, vol. 14 , no. 2, p. 342-355.

DEVELEY, PF. and ENDRIGO, E., 2004. Aves da grande São Paulo: guia de campo. São Paulo: Aves e Fotos Editora. 295 p.

DEVELEY, PF., CAVANA, DD. and PIVELLO, VR., 2005. Aves. In PIVELLO, VR. and VARANDA, EM. (Orgs.). $O$ cerrado Pé-de-Gigante: ecologia e conservação. São Paulo: Secretaria do Meio Ambiente. p. 121-134.

DIAS, MM., 2000. Avifauna das Estações Ecológicas de Jataí e Experimental de Luiz Antônio, São Paulo, Brasil. In SANTOS, JE. and PIRES, JSR. (Eds.). Estação Ecológica de Jataí. São Carlos: Editora Rima. p. 285-301. (vol. 1).

DONATELLI, RJ., FERREIRA, CD., DALBETO, AC. and POSSO, SR., 2007. Análise comparativa da assembléia de aves em dois remanescentes florestais no interior de São Paulo, Brasil. Revista Brasileira de Zoologia, vol. 24, no. 2, p. 362-375.

DURIGAN, G., SARAIVA, IR., GARRIDO, MAO., GARRIDO, LMAG. and PECHE-FILHO, A., 1987. Fitossociologia e evolução da densidade da vegetação de cerrado em Assis, SP. Boletim Técnico do Instituto Florestal, vol. 41, no. 1, p. 59-78.

DURIGAN, G., SIQUEIRA, MF., FRANCO, GADC., BRIDGEWATER, S. and RATTER, JA., 2003. The vegetation of priority areas for cerrado conservation in São Paulo state, Brazil. Edinburgh Journal of Botany, vol. 60, no. 2, p. 217-241.

GRESSLER, DT. and MARINI, MÂ., 2008. Nest, eggs and nestling of the Collared Crescentchest Melanopareia torquata in the Cerrado region, Brazil. Revista Brasileira de Ornitologia, vol. 14 , no. 4, p. 598-600

Instituto Brasileiro do Meio Ambiente e dos Recursos Naturais Renováveis - IBAMA, 2003. Lista das espécies da fauna ameaçadas de extinção. Brasília: Ministério do Meio Ambiente.
International Union for Conservation of Nature and Natural Resources - IUCN, 2007. Red List of Threatened Species. Available from: <http://www.iucnredlist.org $>$. Access in: 02/03/2008.

KANEGAE, MF., TELLES, M. and DAMIANO, RZ., 2008. Primeiro registro fotográfico do desenvolvimento de ninhegos de tapaculo de colarinho. Atualidades Ornitológicas, vol. 141, p. 94-102.

KRÜGEL, MM. and ANJOS, L., 2000. Bird communities in forest remnants in the city of Maringá, Paraná State, Southern Brazil. Ornitologia Neotropical, vol. 11, no. 4, p. 315-330.

LOYOLA, RD., KUBOTA, U. and LEWINSOHN, TM., 2007. Endemic vertebrates are the most effective surrogates for identifying conservation priorities among Brazilian ecoregions. Diversity and Distributions, vol. 13, no. 4, p. 389-396.

MACEDO, RH., 2002. The avifauna: ecology, biogeography, and behavior. In OLIVEIRA, PS. and MARQUIS, RJ. (Eds.). The cerrados of Brazil: ecology and natural history of a neotropical Savanna. New York: Columbia University Press. p. $242-263$.

MACHADO, RB., RAMOS-NETO, MB., HARRIS, MB., LOURIVAL, R. and AGUIAR, LMS., 2004. Análise de lacunas de proteção da biodiversidade no Cerrado. In Anais do IV Congresso Brasileiro de Unidades de Conservação. Curitiba: Fundação O Boticário de Preservação à Natureza. p. 29-38.

MARINI, MÂ., ROBINSON, SK. and HESKE, EJ., 1995. Edge effects on nest predation in the Shawnee National Forest, southern Illinois. Biological Conservation, vol. 74, no. 3, p. 203-213.

MARINI, MÂ., 2001. Effects of forest fragmentation on birds of the cerrado region, Brazil. Bird Conservation International, vol. 11, no. 1 p. 13-25.

MARINI, MÂ. and CAVALCANTI, RB., 1993. Habitat and foraging substrate use of three Basileuterus warblers from central Brazil. Ornitologia Neotropical, vol. 4, no. 2, p. 69-76.

MARINI, MÂ. and GARCIA, FI., 2005. Bird conservation in Brazil. Conservation Biology, vol. 19, no. 3, p. 665-671.

MARINI, MÂ., ROBINSON, SK. and HESKE, EJ., 1995. Edge effects on nest predation in the Shawnee National Forest, Southern Illinois. Biological Conservation, vol. 74, no. 3, p. 203-213.

MARTENSEN, AC., PIMENTEL, RG. and METZGER, JP., 2008. Relative effects of fragment size and connectivity on bird community in the Atlantic Rain Forest: implications for conservation. Biological Conservation, vol. 141, no. 9, p. 2184-2192.

MARZLUFF, JM. and EWING, K., 2001. Restoration of fragmented landscapes for the conservation of birds: a general framework and specific recommendations for urbanizing landscapes. Restoration Ecology, vol. 9, no. 3, p. 280-292.

MEYER DE SCHAUENSEE, R., 1982. A guide to the Birds of South America. Wynnewood: Intercollegiate Press Inc. 498 p.

MOTTA Jr., JC., 1990. Estrutura trófica e composição das avifaunas de três habitats terrestres na região central do Estado de São Paulo. Ararajuba, vol. 1, p. 65-71.

MOTTA Jr., JC. and VASCONCELLOS, LAS., 1996. Levantamento das aves do campus da Universidade Federal de 
São Carlos, Estado de São Paulo, Brasil. In Anais do Seminário Regional de Ecologia. São Carlos: UFSCar. p. 159-171. (vol. 7).

MOTTA Jr., JC., GRANZINOLLI, MAM. and DEVELEY, PF., 2008. Aves da Estação Ecológica de Itirapina, Estado de São Paulo, Brasil. Biota Neotropica, vol. 8, no. 3, p. 207-227.

MURCIA, C., 1996. Forest fragmentation and the pollination of neotropical plants. In SCHELHAS, J. and GREENBERG, RS. (Eds.). Forest Patches in Tropical Landscapes. Washington: Island Press. p. 19-36.

MYERS, N., MITTERMEIER, RA., MITTERMEIER, CG., FONSECA, GAB. and KENT, J., 2000. Biodiversity hotspots for conservation priorities. Nature, vol. 403, p. 853-858.

PALMER, GC., FITZSIMONS, JA., ANTOS, MJ. and WHITE, JG., 2008. Determinants of native avian richness in suburban remnant vegetation: Implications for conservation planning. Biological Conservation, vol. 141, no. 9, p. 2329-2341.

PINTO, MP., DINIZ-FILHO, JAF., BINI, LM., BLAMIRES, D. and RANGEL, TFLVB., 2008. Biodiversity surrogate groups and conservation priority areas: birds of the Brazilian cerrado. Diversity and Distributions, vol. 14, no. 1, p. 78-86.

PIRATELLI, A., SOUSA, SD., CORRÊA, JS., ANDRADE, VA., RIBEIRO, RY., AVELAR, LH. and OLIVEIRA, EF., 2008. Searching for bioindicators of forest fragmentation: passerine birds in the Atlantic forest of southeastern Brazil. Revista Brasileira de Biologia = Brazilian Journal of Biology, vol. 68, no. 2, p. 259-268.

PIZO, MA., 2001. A conservação das aves frugívoras. In ALBUQUERQUE, JLB., CÂNDIDO Jr., JF., STRAUBE, FC. and ROOS, AL. (Eds.). Ornitologia e conservação: da ciência às estratégias. Tubarão: Editora Unisul. p. 49-59.

PRIMAVESI, O., PRIMAVESI, ACPA., PEDROSO, AF., CAMARGO, AC., RASSINI, JB., ROCHA-FILHO, J., OLIVEIRA, GP., CORREA, LA., ARMELIN, MJA., VIEIRA, SR. and DECHEN, SCF., 1999. Microbacia hidrográfica do Ribeirão Canchim: um laboratório real de laboratório ambiental. São Carlos: Embrapa Pecuária Sudeste. 133 p. (boletim de pesquisa no. 5).

SÃO PAULO, 1999. Conhecer para conservar: as unidades de conservação do Estado de São Paulo. São Paulo: Série Probio. $115 \mathrm{p}$.

2008. Fauna ameaçada no Estado de São Paulo. Diário Oficial do Estado de São Paulo, vol. 118, no. 187, 60 p.

SICK, H., 1997. Ornitologia brasileira. In PACHECO, JF. (Coord.). Rio de Janeiro: Nova Fronteira. 912 p.

SILVA, JMC., 1995. Birds of the cerrado region, South America. Steenstrupia, vol. 21, no. 1, p. 69-92.

1997. Endemic bird species and conservation in the Cerrado Region, South America. Biodiversity Conservation, vol. 6 , no. 3, p. 435-450.

SILVA, JMC. and BATES, JM., 2002. Biogeografic patterns and conservation in the South American cerrado: a tropical savanna hotspot. Bioscience, vol. 52, no. 3, p. 225-233.

SOUZA, DGS., 1998. Todas as aves do Brasil: guia de campo para identificação. Feira de Santana: Dall. 350 p.
STOTZ, DF., FITZPATRICK, JW., PARKER III, TA. and MOSKOVITS, DK., 1996. Neotropical birds: ecology and conservation. Chicago: Chicago University Press. 478 p.

STOUFFER, PC. and BIERREGAARD, JR., 1995. Effects of forest fragmentation on understory hummingbirds in Amazonian Brazil. Conservation Biology, vol. 9, no. 5, p. 1085-1091.

TELLES, M. and DIAS, MM., 2010. Bird communities in two fragments of Cerrado in Itirapina, Brazil. Revista Brasileira de Biologia $=$ Brazilian Journal of Biology, in press.

TELLINO Jr., WR., DIAS, MM., AZEVEDO Jr., SM., LYRA-NEVES, RM. and LARRAZÁBAL, MEL., 2005. Estrutura trófica da avifauna na Reserva Estadual de Gurjaú, Zona da Mata Sul, Pernambuco, Brasil. Revista Brasileira de Zoologia, vol. 22, no. 4, p. 962-973.

TUBELIS, DP. and CAVALCANTI, RB., 2000. A comparison of bird communities in natural and disturbed non-wetland open habitats in the cerrado's central region, Brazil. Bird Conservation International, vol. 10, no. 4, p. 331-350.

TUBELIS, DP., COWLING, A. and DONNELLY, C., 2004. Landscape supplementation in adjacent savannas and its implications for the design of corridors for forest birds in the central Cerrado, Brazil. Biological Conservation, vol. 118, no. 3, p. 353-364.

VIELLIARD, JME. and SILVA, WR., 1990. Nova metodologia de levantamento quantitativo e primeiros resultados no interior de São Paulo. In Anais do IV Encontro Nacional de Anilhadores de Aves. Pernambuco: Universidade de Pernambuco. p. 117-151.

WATSON, JEM., WHITTAKER, RJ. and FREUDENBERGER, D., 2005. Bird community responses to habitat fragmentation: how consistent are they across landscapes? Journal of Biogeography, vol. 32, no. 8, p. 1353-1370.

WILLIS, EO., 1979. The composition of avian communities in remanescent woodlots in southern Brazil. Papéis Avulsos de Zoologia, vol. 33, no. 1, p. 1-25.

2004. Birds of a habitat spectrum in the Itirapina savanna, São Paulo, Brazil (1982-2003). Revista Brasileira de Biologia = Brazilian Journal of Biology, vol. 64, no. 4, p. 901-910.

2006. Protected cerrado fragments grow up and lose even metapopulational birds in central São Paulo, Brazil. Revista Brasileira de Biologia = Brazilian Journal of Biology, vol. 66, no. 3, p. 829-837.

WILLIS, EO. and ONIKI, Y., 1987. Invasion of deforested regions of São Paulo State by the picazuro pigeon, Columba picazuro Temminck 1813. Ciência e Cultura, vol. 39, no. 11, p. 1064-1065.

2003. Aves do Estado de São Paulo. Rio Claro: Editora Divisa. 398 p.

ZANETTE, L., DOYLE, P. and TRÉMONT, SM., 2000. Food shortage in small fragments: evidence from an area-sensitive passerine. Ecology, vol. 81, no. 6, p. 1654-1666. 